\title{
Análisis crítico del discurso: la reforma a la educación superior en Chile en medios de comunicación escritos
}

Critical Discourse Analysis: The Higher Education Reform in Chile in Written Media

Análise crítica do discurso: a reforma da Educação Superior no Chile em meios de comunicação escrita

Pablo Abraham Camus* iD orcid.org/0000-0001-6463-3892
Nicolas Ponce** iD orcid.org/0000-0003-0670-9590
Franco Cataldo Contreras*** iD orcid.org/0000-0002-0603-8582
Milenko del Valle Tapia**** iD orcid.org/0000-0003-3739-1001

Artículo de investigación

Revista Colombiana de Educación, N. 75. Segundo semestre de 2018, Bogotá, Colombia

doi: 10.17227/rce.num75-8102

Para citar este artículo: Camus, P., Ponce, N., Cataldo, F. y del Valle, M. (2018). Análisis crítico del discurso: la reforma a la educación superior en Chile en medios de comunicación escritos. Revista Colombiana de Educación, 75, 77-98.

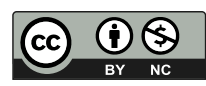

* Doctor en Sociología. Académico Universidad de Antofagasta, Antofagasta, Chile. Correo electrónico: pablo.camus@uantof.cl

** Magíster en Psicología Educacional. Académico Universidad de Antofagasta, Antofagasta, Chile. Correo electrónico: nicolas.ponce@uantof.cl

*** Sociólogo. Académico Universidad de Antofagasta, Antofagasta, Chile. Correo electrónico: franco.cataldo@uantof.cl

**** Magíster en Educación. Mención Investigación Educación. Académico Universidad de Antofagasta Antofagasta, Chile.

Correo electrónico: milenkodelvalle@uantof.c 


\section{Resumen}

El presente artículo da cuenta de una investigación, de carácter cualitativo, que realiza el análisis crítico del discurso (ACD) de los actores involucrados en el debate presente acerca de la propuesta de reforma a la educación superior (RES) que se desarrolla en Chile, y que se expresa a través de los medios de comunicación escritos. Se devela la posición social de los actores en la estructura social o por la influencia que ejercen en la problemática discutida. Además, se identifica el discurso ideológico que subyace al contenido emitido por los medios de comunicación. Los principales resultados dan cuenta de la crítica explícita al proceso de RES por insuficiente, por el distanciamiento de las instituciones con respecto a la realidad social o por la intensificación de los procesos de privatización. Asimismo, se observa la reiteración de algunas estructuras que dan cuenta, de forma crítica, de los elementos que subyacen al proceso de reforma, como son la criminalización del movimiento estudiantil, la identificación del sistema educativo como bien de consumo y el fortalecimiento del sector privado, entre otras.

\section{Palabras clave}

Educación superior; Gobierno; movimiento estudiantil; crónica noticiosa
Keywords

Higher Education; Government; Student Movement; News

Chronicle

\begin{abstract}
This article presents a qualitative research that accounts for the critical discourse analysis (CDA) of the actors involved in the current debate about the proposal for higher education reform (RES) that takes place in Chile, and that is expressed through the written media. The social position of the actors in the social structure or the influence they exert on the problem discussed is revealed. In addition, the underlying ideological discourse in the content issued by the media is identified. The main results account for the explicit criticism of the RES process as insufficient, for the distancing of the institutions with regard to the social reality or for the intensification of the privatization processes. Likewise, we observe the reiteration of some structures that give a critical account of the underlying elements in the reform process, such as the criminalization of the student movement, the identification of the educational system as a consumer good and the strengthening of the private sector, among others.
\end{abstract}

\section{Resumo}

O presente artigo dá conta de uma pesquisa, de caráter qualitativo, que apresenta a análise crítica do discurso (ACD) dos sujeitos envolvidos no debate presente acerca da proposta da reforma à educação superior (RES) no Chile, expressada através dos meios de comunicação escrita. Revela-se a posição social dos atores na estrutura social ou pela influência exercida na problemática discutida. Além disso, identifica-se o discurso ideológico que subjaz ao conteúdo emitido pelos médios de comunicação. As principais descobertas dão conta da crítica explícita ao processo de REs por sua insuficiência, pelo seu distanciamento das instituições em relação à realidade social ou pela intensificação dos processos de privatização. Assim mesmo, observa-se a reiteração de algumas estruturas que evidenciam, de forma crítica, os elementos que subjazem ao processo de reforma, como a criminalização do movimento estudantil, a identificação do sistema educativo como produto de consumo e o fortalecimento do setor privado, entre outros.

\section{Palavras-chave}

educação superior; governo; movimento estudiantil; crónica informativa 


\section{Introducción}

En la coyuntura sociopolítica actual chilena se ha posicionado entre la opinión pública un prolongado debate sobre la reforma a la educación, impulsada por el gobierno de Michelle Bachelet. Hasta el momento se encuentra aprobada una primera fase, circunscrita a la educación primaria y secundaria. Dicha reforma se encuentra vigente desde marzo de 2016, y el proceso de planificación que la constituyó no estuvo exento de críticas provenientes desde distintos sectores de la sociedad.

En el primer semestre del 2016, el debate se desplazó hacia la siguiente fase, que impulsa un cambio significativo del sistema de educación superior. La RES gira alrededor de ocho ejes, a saber: (1) creación de un sistema de educación superior que articule todas las instituciones y organismos gubernamentales relacionados; (2) creación de una subsecretaría de educación superior; (3) fomento a la formación técnico-profesional; (4) creación del Sistema Nacional del Aseguramiento de la Calidad de la Educación Superior; (5) creación de la Superintendencia de Educación Superior; (6) aumento de la regulación a las instituciones organizadas como personas jurídicas sin fines de lucro; (7) régimen de la educación superior estatal; y (8) financiamiento público.

La necesidad de implementar un cambio estructural en el sistema educativo chileno se remonta por lo menos a treinta años. Empero, fue en el año 2011 cuando se logró instalar con fuerza la necesidad de reestructurar el sistema educacional superior (Silva, 2012), bajo la premisa de que exista calidad y gratuidad en la educación ofrecida por los sistemas público y privado, a fin de incidir en la disminución de la brecha socioeconómica y cultural. En este sentido, los medios de comunicación escritos o electrónicos ejercen gran influencia en la vida social de los individuos en el momento de articular los lineamientos de la opinión pública. Esto revela, de manera latente, las distintas formas en que se manifiesta el poder de las élites. Desde esta mirada, Trujillo (2006) sostiene que los procesos de mercantilización de la información no son ajenos a las sutiles influencias de intereses específicos, particulares o colectivos, lo que denota la existencia de poderes que operan tras estos medios de comunicación.

El presente estudio desarrolla un análisis crítico del discurso (ACD) aplicado a crónicas noticiosas obtenidas desde cuatro medios de comunicación escrita, en relación con el debate generado por los distintos actores involucrados en el contexto de la RES, durante el periodo mayo-julio de 2016.

La investigación da cuenta de los matices discursivos que manifiestan los actores involucrados en los debates presentes en los medios de comunicación impresos y en formato digital. Esto devela, por un lado, la posición social de los actores en la estructura social que adscriben

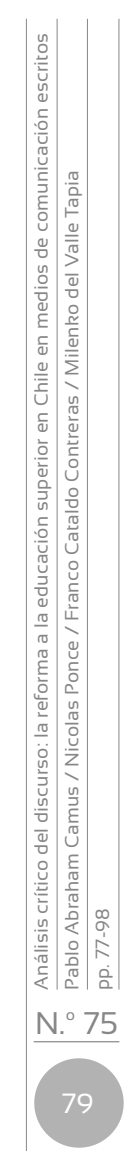


y la influencia que ejercen sobre la problemática discutida; y, por otro, identifica el discurso ideológico que subyace al contenido emitido por los medios, considerando la línea editorial que poseen, el público objetivo al cual se orientan y los grupos de poder que representan.

La pregunta de investigación que servirá tanto de leit motiv como de sistematización del trabajo, es: ¿Cómo se construyen las actorías involucradas en el debate de la REs, según los discursos emitidos en las distintas crónicas noticiosas analizadas?

\section{Conceptos preliminares}

En este apartado se establecen los principios fundamentales desde los cuales se realizará el ACD. La proliferación de discursos sobre la llamada RES requiere hacer converger aquellos instrumentos teóricos y prácticos. En este sentido, se busca descubrir los contenidos transversales y las diferencias en los discursos, y desde ellos derivar las observaciones y comentarios para culminar en el análisis crítico. Desde esta perspectiva, se presentan articuladamente tanto las principales perspectivas sobre el ACD como las producciones teóricas que proceden desde la sociología y la filosofía, que otorgarán mayor riqueza al proceso analítico.

\section{Aproximaciones a los conceptos de texto y discurso}

Un acercamiento inicial al problema objeto de estudio es establecer la conceptualización base para entender los conceptos de texto y discurso.

Para empezar, podemos definir como texto "a todo discurso fijado por la escritura" (Ricoeur, 2001, p. 127). Esta definición contiene el núcleo sustantivo de lo que se entenderá por texto, en cuanto que es el plano objetivo y material de un proceso que adquiere valor hermenéutico, en la medida en que sirve para visibilizar e interpretar las acciones significativas de los sujetos en sociedad. Una segunda afirmación es que "el texto en sí mismo no posee sentido; es solo un mediador y la vía hacia el sentido" (Conde, 2009, p. 34).

En cuanto a la noción de discurso, una definición asertiva es la que entrega Meersohn (2005), quien plantea el discurso como una forma específica del uso del lenguaje, que a su vez contiene un modo particular de interacción social. Así, el discurso se interpreta como un evento comunicativo completo e inserto en una situación social.

En las ciencias sociales, el discurso ha acaparado el interés como objeto de estudio de la filosofía, la lingüística y la sociología, con el fin de ser analizado e interpretado para poder otorgarle sentido. De acuerdo con esta premisa, la definición que construye Conde (2009) resulta idónea en 
el desarrollo de esta investigación, pues ella articula la definición en una connotación práctica que escapa de las conceptualizaciones genéricas del uso coloquial. El discurso se define como equivalente a un conjunto de ideas expresadas oralmente o por escrito, sea con la expresión en una intervención formal, en una conversación o en cualquier otro entorno.

Esta orientación metodológica se aplicará en el análisis de los textos acotados en este trabajo. Así, el estudio y su interpretación relevarán "quién utiliza el lenguaje, cómo lo utiliza, por qué y cuándo lo hace; así como para qué y para quién lo hace" (Conde, 2009, p. 36).

Para articular el análisis se incorpora otro principio que atiende a la indivisibilidad del discurso dentro de un todo mayor, es decir, todo discurso está inserto en un "sistema de discursos", puesto que ellos no se producen aislados, ni existen de forma individual. Por el contrario, todo discurso se produce y desarrolla con relación a otro discurso que alude, que interpela, al que se trata de aproximar o del que se trata de diferenciar (Conde, 2009).

A continuación, se presenta una aproximación al contenido nuclear de este estudio, la crónica noticiosa, desde la noción expuesta de discurso.

\section{La crónica noticiosa como discurso: la perspectiva conceptual de Van Dijk}

La selección de textos recogida y acotada en el tiempo bajo la forma de la noticia -mejor aún, la crónica noticiosa- surge de un acontecimiento que genera y produce interés. En la medida que dicho acontecimiento afecte a una comunidad, a través de reacciones y comentarios, el hecho transitará desde "acontecimiento" hacia "noticia". Al mismo tiempo, se debe tener presente que no todos los acontecimientos logran constituirse como noticia, lo que se explica por el interés que despierta en los distintos centros periodísticos. Una noticia que puede despertar el interés para un diario $x$ y una comunidad $x$, para un diario $z$ y una comunidad $z$ será irrelevante (Saad, 2010).

En esta perspectiva conceptual, resulta necesario precisar una aproximación teórica que refiere a este elemento nuclear del estudio: la crónica noticiosa. En esta línea, Van Dijk (1990), en su obra La noticia como discurso, ha sistematizado el desarrollo que se opera en las formas y estructuras que adquiere el discurso como producción noticiosa. Esta visión se expone de manera sintética a continuación, pues será utilizada en el estudio:

Dentro de un marco socioeconómico y cultural más amplio, este análisis considera el discurso de los medios, y por lo tanto también la noticia, como una forma particular de práctica social, institucional. Esta práctica discursiva de la producción o de la recepción puede analizarse teóricamente en dos componentes principales: un componente 
textual y un componente contextual. El componente textual analiza sistemáticamente las diferentes estructuras del discurso periodístico en diferentes niveles. El componente contextual analiza los factores cognitivos y sociales, las condiciones, los límites o las consecuencias de estas estructuras textuales e, indirectamente, su contexto económico, cultural e histórico. (Van Dijk, 1990, p. 250).

El modelo propuesto por Van Dijk (1990) permite indagar en la relación socio-cognitiva entre el texto y el contexto socioeconómico, vale decir, la manera en que los fabricantes de la noticia y los lectores representan efectivamente los acontecimientos informativos, escriben o leen los textos periodísticos, procesan diferentes textos fuente o participan en los hechos de comunicación.

Este principio será útil, pues posicionará el foco de análisis en las estructuras textuales presentes en las producciones noticiosas, el cual opera en distintos niveles y dimensiones. El autor invita a clarificar las notas generales del discurso y las estructuras específicas que lo diferencian de otros textos de los medios de comunicación. En este sentido, se enfrentan textos buscando distinguir las estructuras textuales. Aquí, el esfuerzo está dado en individualizar el uso de neologismos, de nominalizaciones, la complejidad oracional o el orden de las palabras y cláusulas; todas estas estructuras ofrecen las características del estilo gramatical en los discursos periodísticos. Dichas estructuras, definidas como microestructuras, caracterizan las frases y las relaciones inmediatas entre ellas (Van Dijk, 1983).

El análisis de estas microestructuras hace explícitos tanto el conocimiento que se presupone, las suposiciones subjetivas -esto es, opiniones y actitudes-, como las ideologías sobre las que se basan. Lo anterior se evidencia en las estructuras retóricas y estilísticas presentes en las crónicas noticiosas. Las primeras, obedecen a una función persuasiva, en el sentido de que el mensaje sea más eficaz. Por ejemplo, la prensa utiliza a menudo una serie de recursos retóricos para destacar opiniones, actitudes o emociones: asesinato horrendo, terrible accidente, manifestantes agresivos. Las segundas, se refieren a la elección de las maneras de expresar la información; es decir, se entrega a partir del estilo una valoración positiva o negativa al hecho, por ejemplo a los manifestantes de algún determinado movimiento social, se les puede tildar de encapuchados, criminales o lumpen.

Por último, se han utilizado algunas referencias en el plano de significados globales o totales, es decir, las macro estructuras semánticas, para explicitar la noción familiar del asunto o tema que cubre la información periodística. Estas macro estructuras se explicitan en la información periodística mediante titulares y encabezamientos. En términos del autor: "la definición de la situación tal como la proporciona la macro-estructura temática de un reportaje periodístico puede ser muy diferente de ciertas 
definiciones alternativas" (Van Dijk, 1990, p. 253). Los significados globales necesitan una forma de expresión que obedece a estructuras canónicas establecidas culturalmente. El discurso periodístico en su desarrollo posee una estructura de exposición, nudo y desenlace.

Esta forma de análisis estructural se realiza en función de su contexto de producción, así como en el de comprensión. De esta forma, "deseamos conocer sus funciones específicas sea en la expresión del conocimiento, las creencias, las actitudes e ideologías subyacentes, o como resultados de las restricciones específicas de la fabricación de noticias" (Van Dijk, 1990, p. 255). Al desentrañar las estructuras, se dilucidan también las estrategias y representaciones que desempeñan roles en la interpretación, memorización y reproducción de la información periodística por parte de los lectores. De este modo, lo que es información importante para un lector, puede no serlo para otro, según sus creencias particulares, opiniones, actitudes, valores, modelos, ideologías y objetivos (Van Dijk, 1983). A continuación se expone el modelo metodológico para realizar un ACD.

\section{Análisis crítico del discurso (ACD): la perspectiva de Van Dijk}

El ACD es necesario abordarlo individualizando aspectos de su concepción teórica. En este sentido, los aportes expuestos han creado un horizonte de base para adentrarse en el ACD. Así, los aspectos que se desarrollan son las herramientas que se usan tanto en la recolección de la muestra como en el análisis de los textos seleccionados.

Una aproximación inicial es compartir la posición de Pardo (2007) sobre el ACD, quien sostiene que los datos obtenidos se enmarcan en una perspectiva histórico-social, cognitiva y lingüística que busca generar conciencia sobre un espacio sociocultural. Esta orientación devela tanto las ideologías como las distintas formas y procesos sociales de circulación simbólica del poder en el mundo social, a partir del reconocimiento del carácter consensuado de los discursos, de las diferencias discursivas y de sus usos tapizados por los ejercicios de poder. En otros términos, siguiendo a Van Dijk (1999), este trabajo es un tipo de investigación analítica sobre el discurso que intenta dar cuenta del modo en que el abuso de poder social, el dominio y la desigualdad son practicados y reproducidos -y ocasionalmente combatidos- por los textos y el habla en el contexto social y político.

El autor entiende la noción de poder como "control", puesto que los grupos que tienen (más o menos) poder, serán capaces de controlar (más o menos) en su interés particular los actos y la mente de los miembros de otros grupos. La mente humana controla la acción, por lo que, si un discurso alcanza el objetivo de influir sobre la mentalidad de un grupo, ya sea en sus conocimientos u opiniones, podrá controlar de manera indirecta alguna de sus acciones. En este horizonte de comprensión del ACD, la centralidad está

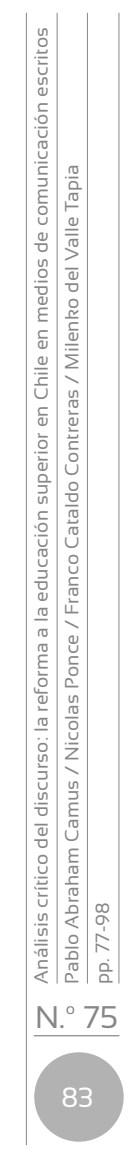


en la explotación de dicho poder y en el dominio que se ejerce, cuando se abusa del control sobre el discurso para controlar las creencias y acciones de la gente en interés de los grupos dominantes (Van Dijk, 1999).

Un paso de profundidad es preguntarse cómo se manifiestan dichas estrategias discursivas de control social/mental. Van Dijk (1999) la responde identificando algunos rasgos característicos: la influencia del contexto y el control de la situación social por los grupos dominantes pueden conducir a modelos contextuales que hacen aparecer su discurso como más creíble, principalmente, mediante la eliminación o el desprestigio de fuentes alternativas de información y de opinión. En síntesis, se tienen los siguientes elementos constitutivos del discurso: (a) macro estructuras semánticas, b) esquemas discursivos (superestructuras, esquemas textuales); y (c) microestructuras sintácticas.

El ACD busca comprender las formas de reproducción del dominio y la desigualdad social que se desprenden del discurso, así como de qué manera estas influyen en la mente del público al que se orientan. No obstante, existen algunas compensaciones "en las sociedades más o menos democráticas, donde diversos grupos compiten por el poder" (Van Dijk, 1999, p. 32).

La complejidad en las relaciones entre discurso y poder radica en que no existe un único grupo que controle de manera hegemónica el discurso público; y si lo hubiese, el discurso controla marginalmente la forma de pensar de los grupos dominados y, en menor medida aún, sus acciones. Es probable que algunos sectores de los grupos de poder muestren solidaridad con grupos dominados y apoyen mediante el discurso público la lucha por la desigualdad; por ello, el ACD penetra con suficiencia la compleja interacción de los grupos dominantes, disidentes, opositores y sus discursos dentro de la sociedad, con el fin de esclarecer las variantes contemporáneas de la desigualdad social (Van Dijk, 1999).

Como último elemento en la arquitectura epistemológica de esta investigación, se recurre a la propuesta del sociólogo francés Pierre Bourdieu, a fin de entender el sistema de relaciones e intereses existentes en el debate sobre REs presentes en las crónicas noticiosas, hecho que se relaciona directamente en lo expuesto sobre el ACD en virtud de esclarecer la complejidad de interacciones de los grupos dominantes, disidentes y opositores.

\section{La teoría de los campos de Pierre Bourdieu}

El núcleo del pensamiento de Bourdieu (1979, 1992, 1997, 2005, 2007, 2012) reside en la relación dialéctica entre los conceptos de campo y habitus. El autor ve a la sociedad como un sistema relacional de diferencias, en el que existen una serie de campos con determinadas reglas de 
juego. La sociedad se constituye mediante espacios de relaciones sociales estructuralmente diferenciados y relativamente autónomos, que son los campos donde los agentes insertan sus trayectorias (Bourdieu, 1997).

El campo es una especie de mercado competitivo en el que se emplean y despliegan tipos de capital: económico, cultural, social y simbólico. La posición que los agentes posean dentro de un determinado campo dependen de la cantidad y el peso relativos del capital propio. En este sentido, Bourdieu es enfático al señalar que el capital simbólico solo existe en la medida en que los otros lo perciben como un valor.

Por otra parte, y siguiendo la línea teórica planteada por Bourdieu, es menester acercarnos al concepto de habitus, que corresponde a la forma en que las estructuras sociales se internalizan en la subjetividad de los agentes sociales. A partir de este concepto, los agentes formarán un conjunto de esquemas prácticos de percepción o una categorización del mundo, sobre lo adecuado-inadecuado, bello-feo, apropiado-inapropiado, y articularán las prácticas y elecciones de los agentes.

Así, el habitus permite explicar por qué las prácticas de los sujetos no pueden comprenderse únicamente en referencia a su posición actual en la estructura social (Bourdieu, 2007). El habitus entendido como principio generador de prácticas, ha sido adquirido durante la "socialización primaria", mediante la familiarización con unas prácticas y espacios que son producidos siguiendo los mismos esquemas y en los que se hayan inscritas las divisiones del mundo social.

Este concepto está estrechamente relacionado con la racionalidad práctica que permite a los sujetos responder con urgencia (aquí y ahora) a partir de los esquemas incorporados socialmente. Si los agentes están inmersos dentro de un juego de apropiación de algún determinado tipo de capital, es que han incorporado ese interés a través de la inmersión en un universo de prácticas que define lo que está en juego. De esta manera, apreciamos el campo como una red de relaciones objetivas entre posiciones, un espacio donde los agentes actúan, pero a su vez están limitados por las mismas reglas que lo conforman.

\section{Aspectos metodológicos de la propuesta}

\section{Enfoque}

Para establecer los matices discursivos presentes en la prensa nacional con relación a la RES, se requiere contar con una arquitectura metodológica que permita descubrir o afinar la pregunta de investigación, a partir de procesos de análisis e interpretación (Valles, 2003). En este sentido, el 
enfoque de investigación de carácter cualitativo intenta comprender las formas discursivas concretas, o meta discursos (Ferrando, Ibañez y Alvira, 2010), de sujetos o grupos presentes en los medios de comunicación, en contexto de la RES de Chile. En este horizonte, se usa una perspectiva hermenéutica que permita establecer una relación de diálogo con respecto al objeto de estudio, que se logra con el uso de las técnicas provenientes del ACD.

Las técnicas de procesamiento de los datos recopilados obedecen al ACD de Van Dijk (1999), donde se entregan insumos metodológicos para la elaboración de matrices de procesamiento de información. Además, para abordar las noticias se procederá a utilizar la matriz propuesta por Browne, Silva y Baessolo (2010), y Browne y Romero (2010), sustentada en el ACD (véase la tabla 1).

Tabla 1. Metodología propuesta para el análisis del discurso en crónicas noticiosas

\begin{tabular}{|c|c|}
\hline \multirow{4}{*}{$\begin{array}{l}\text { 1. Plano significativo/texto } \\
\text { (significados e informaciones } \\
\text { que se extraen del texto) }\end{array}$} & $\begin{array}{l}\text { 1.1. Nivel temático: significados globales (temas } \\
\text { de importancia expuestos en la noticia; el } \\
\text { qué y el cómo y sus interacciones) }\end{array}$ \\
\hline & $\begin{array}{l}\text { 1.2. Nivel de significados locales (el } \\
\text { sentido de las palabras): }\end{array}$ \\
\hline & $\begin{array}{l}\text { a. De carácter implícito o indirecto (presupuestos, } \\
\text { ambigüedades, alusiones, hipérboles, etc.) }\end{array}$ \\
\hline & $\begin{array}{l}\text { b. De carácter explícito o directo (construcciones } \\
\text { lingüísticas expresadas de manera evidente) }\end{array}$ \\
\hline \multirow{2}{*}{$\begin{array}{l}\text { 2.- Plano formal/texto-contexto } \\
\text { (ambiente en el cual se desarrolla } \\
\text { la noticia y sus efectos) }\end{array}$} & $\begin{array}{l}\text { 2.1. Estructuras formales sutiles (tipos de } \\
\text { discursos ideológicos presentes en el texto) }\end{array}$ \\
\hline & $\begin{array}{l}\text { 2.2. Nivel contextual (raíces de } \\
\text { las estructuras mentales) }\end{array}$ \\
\hline
\end{tabular}

Fuente: metodología extraída del modelo basado en el ACD de Browne, Silva y Baessolo (2010), y Browne y Romero (2010).

\section{Unidad de análisis y justificación de la muestra}

La unidad de análisis de este estudio corresponde a la crónica noticiosa presente en un grupo de medios de comunicación escrita que circulan en Chile, con una mirada desde la región de Antofagasta ${ }^{1}$, sobre la producción discursiva en la prensa, con relación al debate sobre la RES.

En la selección de los medios sometidos al ACD, el muestreo aplicó tres criterios: (1) tipo de publicación, impreso o digital; (2) según el tiraje o alcance de público que tengan dichos medios (tanto a nivel nacional como regional); y (3) relevancia social que presentan dichos medios para la población. De esta manera, se opta por elegir cuatro medios noticiosos,

1 Segunda región de Chile, ubicada en el extremo norte del país. Limita al norte con la región de Tarapacá, al sur con la región de Atacama, al este con Bolivia y Argentina, y al oeste con el océano Pacífico. 
asunto que se justifica a través de los datos obtenidos por el portal SimilarWeb² recopilados entre los meses de mayo-julio de 2016, y que se sistematiza a continuación (véase la tabla 2).

Tabla 2. Muestra de los medios escritos utilizados

\begin{tabular}{|c|c|c|c|c|}
\hline Alcance & Formato medio & $\begin{array}{c}\text { Medio } \\
\text { seleccionado }\end{array}$ & $\begin{array}{c}\text { Visitas/ alcance } \\
\text { (mensual) }\end{array}$ & $\begin{array}{c}\text { Tiempo } \\
\text { promedio } \\
\text { de visita }\end{array}$ \\
\hline Nacional & Impreso & LUN & $20,9 \mathrm{M}$ & $7^{\prime} 6^{\prime \prime}$ \\
\hline Regional & Digital & Bío-Bío & $10,4 \mathrm{M}$ & $5^{\prime} 4^{\prime \prime}$ \\
& Impreso & Mercurio de & $262 \mathrm{~K}$ & $6^{\prime} 25^{\prime \prime}$ \\
\hline \multirow{2}{*}{ Digital } & Antofagasta & & \\
\hline & El Nortero & $99,8 \mathrm{~K}$ & $0^{\prime} 44^{\prime \prime}$
\end{tabular}

Fuente: elaboración propia a partir de los datos obtenidos del portal SimilarWeb durante agosto del 2016

Con las premisas de método definidas, se procedió a la recopilación de los artículos noticiosos, publicados entre los meses de mayo-julio de 2016. Se pudo dar cuenta de 91 cuerpos noticiosos, correspondientes a los distintos medios elegidos: Las Últimas Noticias (LUN) ${ }^{3}=36$; Portal Web de noticias Bío-Bío $=50 ;$ El Nortero $=1 ;$ El Mercurio de Antofagasta $=4$. Luego, se observó en qué mes se concentraba la mayor cantidad de producción noticiosa alusiva al debate generado por la reforma de educación superior, a fin de concentrar de mejor manera los esfuerzos aplicados en la matriz de ACD. La muestra quedó configurada de la siguiente manera: Bío-Bío = 18; LUN = 10 y El Mercurio de Antofagasta = 1; en total fueron 29 corpus noticiosos sometidos a análisis.

Cabe anotar que el portal noticioso El Nortero durante el mes de julio no realizó ninguna publicación informativa alusiva al movimiento estudiantil o la RES, por lo que finalmente se marginó de la muestra.

Posteriormente, la información clasificada se cruzó con las perspectivas teóricas elegidas, a fin de poder otorgar un sentido crítico y reflexivo sobre la problemática tratada. A continuación, se desarrolla un ejemplo elocuente del análisis realizado mediante la matriz de ACD. En primera instancia se muestra la noticia y luego, la matriz de procesamiento respectiva (véase la tabla 3).

2 Dicho portal realiza estadísticas sobre cualquier sitio web, tomando en consideración el total de visitas, la clasificación a nivel nacional, el tráfico proveniente de distintos dispositivos (PC o móvil) y el tiempo promedio que los usuarios permanecen consumiendo dichos contenidos. No obstante, se acotan las variables a efectos de los objetivos de este estudio. Más información en: https://www.similarweb.com/.

3 Se optó por Las Últimas Noticias (LUN) ya que tanto en el informe de SimilarWeb como en el Estudio Lectoría Gran Santiago, de fuente ipsos, se posiciona como el más leído, tanto en formato impreso como digital. Informe recuperado el 25 de agosto de 2016 y disponible en línea en: http://www.elmercuriomediacenter.cl/wp-content/uploads/estudio-de-lector\%C3\%8da-a-Marzo-20161.pdf. 
Tabla 3. Análisis del primer ejemplo, utilizando una noticia del portal Bío-Bío (05/07/16)

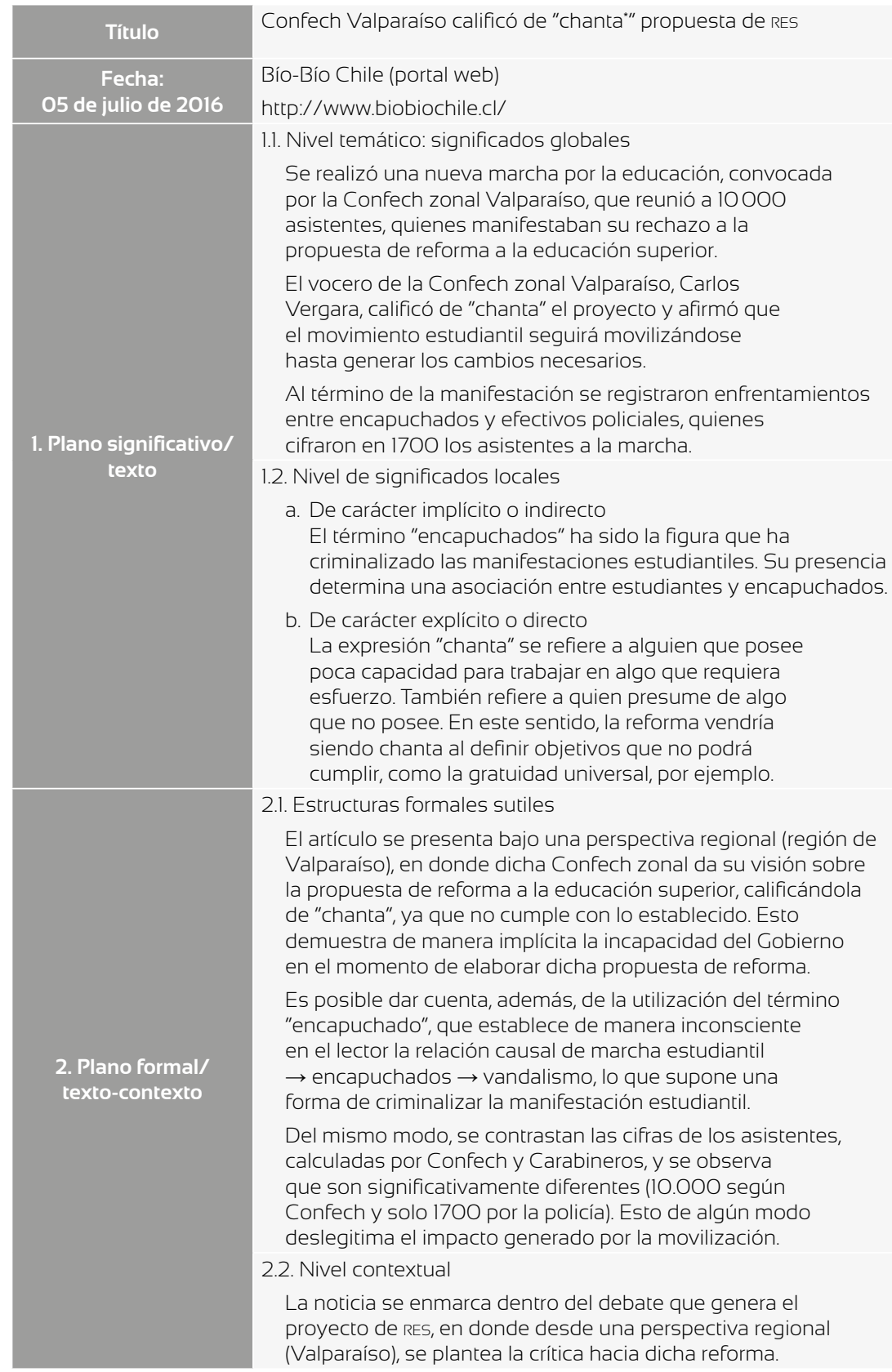

Fuente: elaboración propia

* Referido a una persona que suele actuar de manera fraudulenta y tiene poca credibilidad. Véase Academia Chilena de la Lengua (2010). 


\section{Resultados}

Luego de realizada la recopilación de la prensa nacional, acotada al mes de julio de 2016 y referida a la RES y al movimiento estudiantil, se constituyen 29 corpus noticiosos en los medios escritos seleccionados (véase la tabla 4).

Tabla 4. Sistematización de medios de comunicación escritos utilizados en la investigación

\begin{tabular}{c|c|c|}
\hline Medio escrito & Total corpus & Sección temática \\
\hline LuN & 10 noticias & Política: 3; El día: 7 \\
Bío-Bío Chile & 18 noticias & Nacional \\
\hline El Mercurio de Antofagasta & 1 noticia & Sección: Chile \\
\hline
\end{tabular}

Fuente: elaboración propia

Los resultados se presentan, en primera instancia, desde un plano general y luego, en las dos dimensiones existentes en la matriz analítica, es decir, el plano significado/texto y el plano formal/texto-contexto.

En relación con los resultados generales que se extraen del análisis total de los artículos recopilados, se observa que, a pesar de las pretensiones de entregar una perspectiva regionalista en el estudio, el cubrimiento mediático local sobre la RES en el mes de julio de 2016 fue bajo; solo EI Mercurio de Antofagasta publicó una noticia. Esta situación reflejaría la lógica de centralización, en la cual los debates políticos se concentran, en primer lugar, en la región Metropolitana, luego en otras metrópolis, como Valparaíso y Concepción. En el posterior cruce teórico, se profundizará la reflexión, a fin de entregar una explicación al fenómeno regional.

Como se ha subrayado, en este tipo de análisis es usual el uso de figuras retóricas. En el caso de LuN existe un amplio uso de estas en sus enunciados, especialmente para referirse a las acciones del movimiento estudiantil y para caracterizarlas de manera sarcástica. En un sentido contrario, el portal Bío-Bío utiliza un lenguaje más técnico y formal. En lo que respecta el $A C D$, según la matriz propuesta por Browne, Silva y Baessolo (2010), y Browne y Romero (2010), se articulan los principales resultados según sus dimensiones, a saber:

\section{Plano significado/texto}

\section{Nivel temático}

En términos generales, en los medios sometidos a ACD, se pueden observar algunos contenidos que refieren, principalmente, a orientar la crítica -tanto manifiesta, como latente- hacia el Gobierno y el manejo que les ha dado 
a las demandas generadas por el movimiento estudiantil, y su explícita postura en contra de la institucionalización de la RES. Dichas críticas provienen principalmente desde el mundo académico y universitario; se individualizan tres aspectos principales:

- La reforma se presenta como insuficiente. Tanto el campo académico como el universitario coinciden en que ella no contribuye a solucionar los problemas existentes en el sistema educativo, como la gratuidad universal o el crédito con aval del Estado (CAE). Es un cambio que no resuelve los problemas de fondo.

- Distanciamiento del Gobierno de la realidad social. Este guarda directa relación con lo que acusan los actores, afirmando que existe un distanciamiento evidente del Gobierno con respecto a la realidad social, no solo en materia educacional sino en otros conflictos de igual relevancia, como las pensiones y el medioambiente. Todo incide en la actitud negativa que se genera desde las masas y el mundo académico/ universitario, frente a la ineficiencia mostrada por el poder ejecutivo.

- La reforma fomenta la privatización. Este aspecto también se construye desde los actores académicos ${ }^{4}$ y universitarios, quienes afirman que en la reforma subyace el fomento a la privatización en desmedro de la educación pública. Se acusa al Gobierno de beneficiar al sector privado.

\section{Nivel de significados locales}

De carácter implícito o indirecto. La mayoría de los discursos que construyen las actorías, se realizan en tres vertientes principales y todas con una carga negativa, la que se esconden detrás de enunciados indirectos, aunque bastante elocuentes, a saber: Primero, se construyen críticas a la reforma, situándola como ineficaz por parte de distintos actores entrevistados, cuyo discurso es explicitado en los enunciados de los editores, a través de figuras retóricas. Segundo, se criminaliza a los estudiantes movilizados de manera tangencial en los testimonios expresados por distintos entrevistados. Y tercero, críticas directas a la gestión del gobierno en el manejo del conflicto estudiantil que trae consigo la RES. Es preciso situar en el contexto los distintos enunciados, a fin de lograr explicitar dichas construcciones negativas. En esta línea destaca el siguiente ejemplo:

Título: Vicepresidente de Cruch: "Eyzaguirre explicitó que el CAE continuará de por vida".

4 El rol de los académicos para esta investigación queda en cuestiones netamente retóricas, ya que a partir de sus discursos presentes en las crónicas, se realiza la construcción de la actoría del Gobierno. 
"[...] el ministro Eyzaguirre explicitó que el crédito con aval del Estado, continuará de por vida", incluso para universidades que podrán fijar libremente sus aranceles. (Bío-Bío Chile, 3 de julio de 2016).

El ejemplo da cuenta de cómo se realiza la construcción del rol del Gobierno en la resolución de las demandas generadas por el movimiento estudiantil y que la reforma no contempla. En este sentido, se muestra una actitud reacia por parte del Gobierno en avanzar hacia la gratuidad universal, descartando categóricamente la eliminación del CAE. En otro de los artículos sometidos a análisis sorprende que la Secretaría de Gobierno caracterice este crédito como un beneficio para los estudiantes, cuando es un hecho innegable el endeudamiento que produce en miles de familias y estudiantes.

De carácter explícito o directo. En este tópico, los discursos se articulan con un sentido explícito que no permite la interpretación ambigua por parte del lector.

Título: Marcha estudiantil duró un suspiro.

"Me carga que marchen, porque no los pescan. Apenas un veinte por ciento de ellos sabe por qué pelea, el resto viene a puro lesear"s". (LUN, 29 de julio de 2016).

El testimonio registrado de la locataria, al utilizar la figura "a puro lesear", da cuenta de que no existe conciencia sobre lo que manifiestan los estudiantes, más bien es una actividad de ocio que no posee un sustrato ideológico definido.

\section{Plano formal/texto-contexto}

Estructuras formales sutiles. Se refiere a las estructuras que inciden en el plano psicológico de los receptores, manifestando las dinámicas ideológicas presentes de manera tácita en el discurso. Mediante distintas técnicas informativas, tales como entrevistas o crónicas, se realiza la construcción de las distintas actorías presentes en el debate sobre la reforma a la educación superior. En este sentido, las estructuras que más se repiten son: (1) insuficiencia de la reforma; (2) ineficacia del Gobierno; (3) criminalización al movimiento estudiantil; (4) sátira a las acciones realizadas por el movimiento estudiantil (criminalización simbólica en LUN); (5) movimiento estudiantil como actor que genera propuestas (BíoBío Chile); (6) sistema educativo como bien de consumo; (7) el Gobierno opera a favor del sector privado.

5 No hacer nada provechoso. Véase Academia Chilena de la Lengua (2010). 
Se puede constar que la construcción de las actorías se configura en una lógica de antagonismo, donde finalmente los actores resultantes son el Gobierno y el movimiento estudiantil. La construcción que se realiza del Gobierno, mediante estructuras formales sutiles, supone situar al poder ejecutivo como un actor que ha sido superado por la coyuntura social, incapaz de dar respuesta a las problemáticas planteadas por los movimientos sociales y en particular, del estudiantil.

El movimiento estudiantil, por otro lado, presenta una construcción un tanto ambigua, si se toman como referencia los dos medios escritos de alcance nacional. Por un lado, se criminaliza al movimiento y las acciones que realiza para manifestar su rechazo a la reforma, utilizando declaraciones de distintos actores que reafirman como negativas las acciones Ilevadas a cabo por los estudiantes. Esto lo que en el receptor se traduce en una construcción basada en una percepción negativa frente a los estudiantes. La ambigüedad a la que se hace mención radica en mostrar al movimiento estudiantil como respetuoso de la institucionalidad-solicita permisos a la Intendencia para realizar marchas- o en la contrapropuesta a la reforma que desarrollaron. Sin duda, esa ambigüedad es netamente analítica, dado que en la práctica el discurso varía considerablemente entre Lun y Biobío; los primeros criminalizan y caricaturizan al movimiento estudiantil en mayor medida.

Nivel contextual. En este nivel se logra dilucidar el problema en el cual los receptores articulan los esquemas cognitivos que les permiten interpretar los corpus noticiosos, en contraste con el discurso articulado por las respectivas líneas editoriales de los medios escritos sometidos a análisis. De esta manera, se sitúa una construcción negativa del Gobierno y el movimiento estudiantil, en la cual se ve un conflicto de más de diez años, en el que ninguno de los bandos ha abandonado sus posiciones de intransigencia. La extensión del conflicto social origina un imaginario en el receptor de noticias, en el cual los distintos Gobiernos no han podido hacer frente a las demandas del movimiento estudiantil. Asimismo, los medios de comunicación, lo muestran como impetuoso, al articular las manifestaciones con los hechos de violencia aislados que han ocurrido.

Si bien hubo un periodo (2011-2012) en que la ciudadanía apoyaba transversalmente las demandas generadas por el movimiento estudiantil, la ineficacia mostrada por los distintos Gobiernos (Sebastián Piñera y Michelle Bachelet) ha provocado que el mismo movimiento comience a resentirse y desgastarse, así como el apoyo ciudadano. El rol de los medios de comunicación ha sido fundamental para transmitir la criminalización hacia el movimiento estudiantil, a través de los distintos formatos que poseen, lo que se traduce en una percepción cada vez más negativa hacia la lucha de los estudiantes. Este asunto se replica de manera similar con el Gobierno, donde los medios de comunicación manifiestan constantes críticas a la gestión política del conflicto. 
En síntesis, la construcción de las actorías evidencian dos lógicas antagónicas o contrapuestas: Gobierno y movimiento estudiantil, ambos enfrentados en posicionar su postura con relación a la reforma. La criminalización del movimiento estudiantil y la caracterización de ineficacia e inoperancia del Gobierno frente al manejo político del conflicto social marcan la tónica de la construcción de actorías durante el periodo en estudio.

\section{Conclusiones}

A continuación, se expone un conjunto de conclusiones, cuya arquitectura se organiza desde tres niveles analíticos: conclusiones generales, conclusiones desde el ACD y cruce teórico con el planteamiento de Pierre Bourdieu.

\section{Conclusiones generales}

Luego de someter a análisis los 29 corpus noticiosos -y de elegir dos de ellos para probar las afirmaciones- se constata una primera tendencia de tipo territorial, en la que se realiza la construcción de las principales actorías que confluyen en la coyuntura del debate sobre la RES.

La construcción de los discursos de las actorías se realiza bajo una lógica de centralización-extendida, la cual se concentra en la zona central del país y, de manera esporádica, en otras regiones de esa zona. Esto incide a la vez en un determinismo que hegemoniza las impresiones que han surgido a través del desarrollo del debate, sobre la suficiencia de la reforma impulsada por el Gobierno de Bachelet.

Por otra parte, es Ilamativo que no exista mayor cubrimiento -al menos en la región de Antofagasta- sobre esta coyuntura, dada la existencia de dos universidades miembros del Consejo de Rectores y otras instituciones de este nivel educativo.

Es interesante observar que, de los medios de alcance nacional analizados, Lun y el portal Bío-Bío, solo el último destina mayor cobertura a las reacciones de que ha generado la propuesta de reforma en las regiones. Durante el mes de julio, LUN no registró ninguna de las manifestaciones que han tenido lugar a lo largo del territorio, lo que sorprende dado el alcance y la cantidad de potenciales lectores a los que está expuesto.

La aproximación anterior entrega el primer atisbo sobre la construcción de las actorías, donde la perspectiva centralizada reproduce la lógica que va desde el centro hacia el resto del país.

En un segundo momento, en los corpus analizados se constata que son dos los principales actores (macro actores) en los discursos: el Gobierno y el movimiento estudiantil. Respecto al primero, las crónicas y entrevistas recopiladas lo presentan -ya sean sus acciones y reacciones o 
la gestión de las manifestaciones- como un actor ineficaz cuando se trata de dar respuesta a las problemáticas/necesidades reales que existen en el mundo social, así como ineficiente en la gestión del conflicto estudiantil y por su radicalidad al no realizar modificaciones al proyecto de reforma.

La construcción negativa orientada hacia el Gobierno posee un asidero ideológico de grupos económicos ligados a la propiedad de dichos medios, lo que claramente se expresa en la construcción discursiva presente en las crónicas noticiosas. El caso de LUN es más evidente, dado que el periódico pertenece a El Mercurio S.A.P (al igual que El Mercurio de Antofagasta), empresas de propiedad de Agustín Edwards Eastman, empresario ligado a sectores conservadores.

El portal Bío-Bío pertenece al consorcio de comunicaciones BíoBío Comunicaciones, de propiedad de la familia Mosciatti-Olivieri, cuyo actual dueño, el abogado Tomás Mosciatti, ha tenido incontables discursos críticos hacia la gestión del Gobierno de Bachelet, insinuando incluso que era probable que renunciara a su cargo, dada la magnitud de los distintos conflictos sociales.

Considerando estos alcances, no es extraño que la construcción que se realice sobre los dos principales actores en los medios no varíe en demasía. En Lun la imagen del Gobierno se construye como ineficiente para sortear los obstáculos que suponen los movimientos sociales, e inoperantes para resolver las demandas que la realidad social exige, y criminaliza al movimiento estudiantil en las acciones que realiza a fin de oponerse a la propuesta de reforma.

El caso de Bío-Bío difiere levemente, si bien las críticas hacia la gestión del Gobierno aparecen en la totalidad de los discursos emitidos, la construcción que se realiza del movimiento estudiantil difiere de la del discurso de LUN: solo con excepciones se criminalizan algunas acciones particulares en las marchas, explicitando siempre en las crónicas que las manifestaciones siguen los conductos institucionales, como permisos de Intendencia y reuniones con las instancias de seguridad. También, la actoría movimiento estudiantil se describe como un conglomerado social organizado, capaz de realizar una contrapropuesta a la reforma impulsada por el Gobierno.

\section{Conclusiones a partir de la propuesta teórica de Van Dijk}

Considerando la matriz de ACD con relación al nivel temático, los discursos se orientan críticamente a la gestión del Gobierno y a la criminalización del Movimiento Estudiantil. Estos discursos se realizan de una manera indirecta, vale decir, mediante otros actores que, a través de las entrevistas realizadas, construyen indirectamente a los dos actores relevantes en esta 
investigación. Esto implica que, si bien, en muchas ocasiones se expone la opinión de académicos, políticos o ciudadanos, la configuración discursiva que adopta la entrevista se orienta a realizar la construcción de la actoría del Gobierno y el movimiento estudiantil, lo que influye directamente en la percepción que tendrá el lector y, por consiguiente, en la manera como orientará sus cursos de acción/opinión.

En este sentido, una gran parte del punto de vista oculto, de las opiniones tácitas o de las ideologías usualmente negadas por la prensa pueden inferirse a partir de estas descripciones e identificaciones del léxico de los grupos sociales y de sus miembros (Van Dijk, 1990). El caso más claro donde se manifiesta lo anterior es el de las crónicas de LUN, en las que se utiliza una serie de apelativos para criminalizar al movimiento estudiantil, a modo de ejemplo: "festival de unos", para referirse a la reprobación masiva de estudiantes de la Universidad Alberto Hurtado, que se encontraban paralizados, banalizando además la situación que enfrentaban más de 1200 estudiantes; "a puro lesear", con relación a la opinión de una locataria sobre la convocatoria a una marcha no autorizada; o "duró un suspiro", sobre la misma marcha, que fue duramente reprimida al no contar con el permiso de la Intendencia.

Estos apelativos poseen la intención persuasiva de que el autor da cuenta:

El discurso no es -ni en principio ni en su intención- persuasivo, bien puede tener una dimensión persuasiva en un sentido más indirecto: incluso aunque no argumente a favor de una posición u opinión, ciertamente las presupone, por la definición de su inserción social y, por lo tanto, ideológica. (Van Dijk, 1990).

Finalmente, sobre los significados locales implícitos más recurrentes, destacan los de la insuficiencia del Gobierno para enfrentar el conflicto estudiantil; la nula voluntad política para abolir el CAE; acusaciones contra el Gobierno de impulsar una reforma que favorece al sector privado; "encapuchados" ", "ocupación" o "toma"; "beneficio" -personeros del Gobierno que se refieren al CAE como beneficio- $y$ "criminalización". Con relación a los significados locales explícitos, destacan: "improvisada"; "educación como bien de consumo"; "abusos"; "reforma chanta" y "a puro lesear".

A través de los recursos retóricos que han sido expuestos, se impone una construcción del mundo social que expresa los intereses de las élites económicas y políticas, articulando a las principales actorías según su conveniencia. Así logran condicionar y predisponer a los lectores a tomar una posición y actitud negativa hacia el movimiento estudiantil y el Gobierno.

6 Persona, por lo común joven, que participa en protestas con rostro cubierto, generalmente implicándose en actos de violencia. Véase Academia Chilena de la Lengua (2010). 


\section{Conclusiones desde la teoría de los campos de Pierre Bourdieu}

En este tercer nivel de análisis bajo la teoría de los campos sociales de Bourdieu, se tratará de comprender la lógica de relaciones existentes entre los distintos actores. En este punto, existen intereses que se contraponen a fin de mantener el statu quo. Aquí las construcciones discursivas tienen como fin ulterior manifestar los beneficios económicos y simbólicos de la clase dominante.

Las relaciones que se establecen en torno al debate de la reforma enfrentan a diversos actores, quienes a través de distintos medios y fines diferenciados, según su posición en la estructura del campo de fuerzas (opinión pública), intentarán transformar o conservar la estructura social, en virtud de los intereses subjetivos de cada grupo.

En el campo social de la opinión pública convergen diversos capitales en sus distintas variantes: económica, cultural, simbólica y social. En este sentido, en la conformación de la opinión pública se aprecia una lógica de dominación, que se traduce en el ejercicio de violencia simbólica por parte de la prensa a las dos principales actorías que se desprenden del ACD: el movimiento estudiantil y el Gobierno.

En el ACD quedaron definidos los intereses contrapuestos entre esas dos grandes actorías. No obstante, en este nivel analítico hay que observar la dinámica de relaciones que existe sobre el debate, entre los medios de comunicación escritos y los lectores, elementos constitutivos de la opinión pública, donde se expresa bajo el campo social y el juego (o enjeux) que tiene lugar dentro de dicho campo. En este sentido, el concepto de habitus emerge de manera oportuna para dar cuenta de cómo se expresa la violencia simbólica en esta dinámica de interacción entre medios de comunicación y lectores. El discurso emitido a través de estos medios reproduce la ideología de las élites chilenas, que poseen una resistencia evidente a las transformaciones sociales, por lo que mediante las crónicas noticiosas generan habitus en los lectores, que inciden de manera latente en la percepción y la construcción de la realidad social. Así moldean la opinión pública y orientan los posibles cursos de acción que realicen los individuos, como lectores y consumidores de dichas crónicas noticiosas.

De esta manera, se encuentran en este plano analítico dos niveles de actorías. El lector y los medios de comunicación, vistos como actores, se encuentran sobre las dos "micro actorías" sometidas a análisis en este artículo (Gobierno y movimiento estudiantil), dado que en este "macro nivel" se constituye la opinión pública, lo que confluye directamente en los cursos de acción que toman las "micro actorías". El habitus generado mediante la absorción del discurso por parte de los lectores y el posterior 
efecto en la opinión pública incide en gran medida en algunas acciones que puedan tomar el movimiento estudiantil y el Gobierno en la solución de dichos conflictos.

\section{Referencias}

Academia Chilena de la Lengua. (2010). Diccionario de uso del español de Chile. Santiago de Chile: MN Editorial.

Bourdieu, P. (1979). Los tres estados del capital cultural. Sociológica, UAM- Azcapotzalco, México, 5, 11-17.

Bourdieu, P. (1992). Las reglas del arte. Génesis y estructura del campo literario. Barcelona: Anagrama.

Bourdieu, P. (1997). Razones prácticas. Sobre la teoría de la acción. Barcelona: Anagrama.

Bourdieu, P. y Wacquant, L. (2005). Una invitación a la sociología reflexiva. Buenos Aires: Siglo xxı Editores Argentina.

Bourdieu, P. (2007). El sentido práctico. Madrid: Siglo xxı.

Bourdieu, P. (2012). Intelectuales, política y poder. Madrid: Eudeba.

Browne, R. y Romero, P. (2010). Análisis crítico del discurso de la representación boliviana en las noticias de prensa diaria de cobertura nacional: el caso del Mercurio y La Tercera. Polis, 233-249.

Browne, R., Silva, V. y Baessolo, R. (2010). Periodismo intercultural: representación peruana y boliviana en la prensa chilena. Polis, 85-93.

Conde, F. (2009). Análisis sociológico del sistema de discursos. Cuadernos metodológicos. Madrid: Centro de Investigaciones Sociológicas.

Ferrando, M., Ibañez, J. y Alvira, F. (2010). El análisis de la realidad social. Métodos y técnicas de investigación. Madrid: Ciencias Sociales Alianza Editorial.

Meersohn, C. (2005). Introducción a Teun van Dijk: Análisis del discurso. Cinta de Moebio, 24, 288-302.

Pardo, N. G. (2007). Cómo hacer análisis crítico del discurso. Una perspectiva latinoamericana. Bogotá: Universidad Nacional de Colombia.

Ricoeur, P. (2001). Del texto a la acción. Buenos Aires: Fondo de Cultura Económica de Argentina.

Saad, A. (2010). Una aproximación a la evolución del discurso periodístico del periodismo, la noticia y la subjetividad. Encuentros, 21-30.

Silva, B. (2012). La "Revolución Pingüina" y el cambio cultural en Chile. Recuperado de http://www.archivochile.com/carril_c/cc2012/ cc2012-062.pdf 
Trujillo, S. (2006). Espejos rotos: La intrincada relación de las mujeres y el periodismo impreso en Guatemala. Guatemala: Editorial de Ciencias Sociales.

Valles, M. (2003). Técnicas cualitativas de investigación social. Madrid: Síntesis.

Van Dijk, T. (1983). Estructuras textuales de las noticias de prensa. Análisi. Quaderns de comunicació i cultura, 77-105.

Van Dijk, T. (1990). La noticia como discurso. Barcelona: Paidós.

Van Dijk, T. (1999). El análisis crítico del discurso (pp. 23-36). (Barcelona: Anthropos. Recuperado de www.discursos.org/oldarticles/El\%20 análisis\%20crítico\%20del\%20discurso.pdf 